\title{
ASSUMPTIONS FOR THE FIBER MOVEMENT MODEL IN THE DRAWING MECHANISM FIELD IN MODIFIED REGULATION SYSTEMS
}

\author{
Marek Idzik \\ Technical University of Łódź, Department of Material, Commodity Sciences and Textile Metrology, ul. Żeromskiego 116, 90-924 Łódź, Poland \\ E-mail: marek.idzik@p.lodz.pl
}

\begin{abstract}
:
The paper presents assumptions for the fiber movement model in the drawing mechanism field, aiming at establishing a regulation equation with a modified operation algorithm of the short-term draft regulator of slivers made of staple fibers. The model takes into account the sliver retardation phenomena and makes it possible to increase the evenness of slivers feeding successive machines in the technological process. As a result of this, the evenness of yarn will be increased contributing to an improvement in the quality of flat textile fabrics. This model, despite simplifying assumptions, sufficiently precisely presents the phenomenon of reducing the effect of drawing waves on the distribution of the linear mass of the fiber stream.
\end{abstract}

\section{Keywords:}

draw regulation, sliver, process modeling

\section{Introduction}

The formation of staple fibers into spinning intermediate products and yarn requires knowledge, experience, and appropriate machines. Technological spinning processes are relatively long, and possible faults and disturbances in the fiber stream in any processing stage will be reflected in the yarn made. Staple fibers processed in spinning frames are subjected to the action of many drawing zones. Their main task consists of thinning the fiber stream and straightening and mixing the fibers. However, in the drawing zones, the levelness of fiber distribution in the stream is disturbed and consequently the unevenness of intermediate products and yarn increases. The fiber contact with rotating machine elements results in the formation of drawing waves in the fiber stream. These waves destructively affect the fiber distribution in yarn [1, 2] and consequently lead to the unevenness of flat textile fabrics. This phenomenon can be and even should be reduced in order to improve the yarn quality [3].

Currently, when testing spinning processes, an experimental mathematical model was created on the basis of multidimensional regression analysis to select drawing parameters that influence the non-uniformity of the fiber assembly mass coming out from the drafting system [1]. The new research trend of the drawing process was presented considering the problem of fiber assembly drawing in the drafting zone in terms of questions connected with the analysis of dynamic systems. The main objective is to obtain expressions in the form of transmittance for dynamic relations between the input and quantities of the drafting arrangement.

There are two basic methods of reducing the mass unevenness of linear textile products as follows: doubling the spinning intermediate products and automatic regulation of the drawing process. Doubling effectively reduces the drawing waves, especially those that have relatively high amplitudes. On the other hand, in the case of small variations in linear density, doubling not only fails to decrease irregularities but also increases them as a result of the operation of drawing mechanisms of successive machines in the technological process. At the same time, upon shortening the technological process the role of the doubling process decreases. Thus, there remains the automatic draft regulation, most often used for slivers, whose task consists of eliminating the drawing waves in the fiber stream by the realization of appropriate algorithm action on the control signals forced by signals from measuring systems $[4,5]$.

\section{Automatic draft regulation - analysis of the model}

The automatic draft regulation should be carried out according to the regulation equation for determining the dependence of momentary draft in the drawing mechanism on the variations in sliver tenacity recorded by a measuring device [6]. The regulation equation, especially that for the short-term regulators, developed only on the basis of the phenomena occurring in the draft mechanism, will not only allow one to obtain a sliver with the best levelness of fiber distribution [7-10] but also constitutes a starting point for the modification of the algorithm on the basis of the phenomena occurring in the sliver after the short-term regulation process.

In the model for the cotton-type fibers to be developed, it was assumed that all the fibers in the drawing field move with feeding rate $V_{1}$ up to the moment when their front ends are 
grabbed by the delivery rollers. The fibers, whose front ends have moved to the delivery roller clamps, from that moment move with delivery rate $V_{2}$. The scheme of fiber drawing can be presented in the space-time system in the form of the movement model of fibers with the same length I (Figure 1).

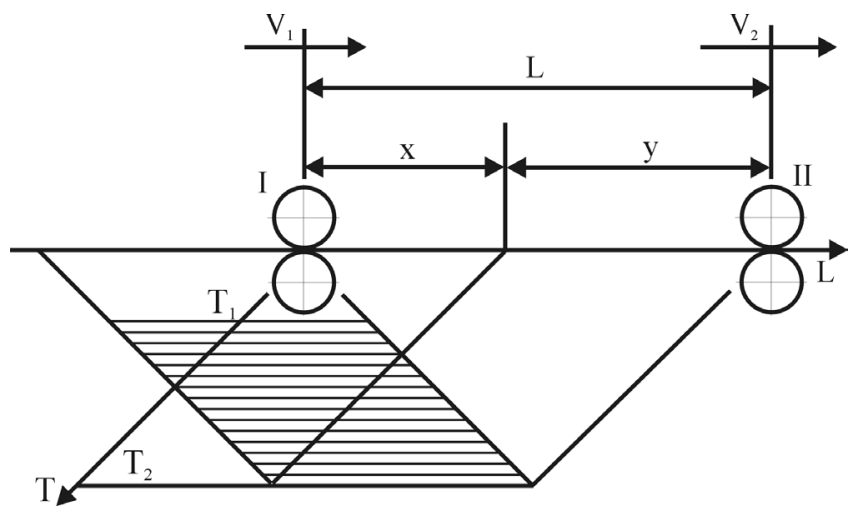

Figure 1. Space-time model of fiber movement

In the sliver cross-section with coordinate $x$ in the drawing field in any moment $t$ are the front ends that have entered the feeding rollers grip in moment $t-T$. If the front ends of fibers in the cross-section $x$ have passed through feeding rollers at time from $T_{1}$ to $T_{2}$, then the number of fibers in the cross-section $x$ in the moment $t$ can be determined by the equation:

$$
m(x ; t)=\int_{T_{1}}^{T_{2}} n_{1}(0 ; t-T) \cdot V_{1} \cdot d T
$$

where $n_{1}(0 ; t-T)$ is the number of the fiber front ends in the feeding rollers grip at time $t-T$;

$$
T_{1}=\frac{x}{V_{1}} \quad T_{2}=\frac{x+l}{V_{1}}
$$

It is assumed that in any cross-section of the feeding product, the distribution of fiber length is constant and that the number of the fiber front ends per the product length unit, i.e. the fiber front ends density changes according to the harmonic curve:

$$
n_{1}(0 ; t)=n_{1}\left[1+\alpha \cos \left(\frac{2 \pi V_{1}}{\lambda_{1}} \cdot t\right)\right]
$$

where $n_{1}$ is the average density of the fiber front ends in the product; $n_{1} \alpha$ is the amplitude of changes in the fiber front ends in the product; and $\lambda_{1}$ is the length of the wave of changes in the fiber front ends density.

Changes in the linear density of a spinning product consist of purely periodic and accidental changes. The principles of draft regulation established for the case of purely periodic changes can then be adapted to the real, more complex conditions. Once relationships (2) and (3) are taken into account in equation (1) and integrated, we have:

$$
m(x ; t)=n_{1} \cdot l+\frac{n_{1} \cdot \alpha \cdot \lambda_{1}}{2} \cdot \sin \frac{\beta+\gamma}{2} \cdot \cos \left(\alpha-\frac{\beta+\gamma}{2}\right)
$$

where

$$
\alpha=\frac{2 \pi V_{1}}{\lambda_{1}} \cdot t \quad \beta=\frac{2 \pi(x+l)}{\lambda_{1}} \quad \gamma=\frac{2 \pi x}{\lambda_{1}}
$$

For $x=0$, we have $y=0$ and $\beta=\frac{2 \pi l}{\lambda_{1}}$, and equation (4) assumes the following form:

$$
m(0 ; t)=m_{1}\left\{1+\alpha \cdot A \cos \left[\frac{2 \pi}{\lambda_{1}} \cdot\left(V_{1} t-L_{\varphi}\right)\right]\right\}
$$

where $m_{1}$ is the average fiber density in the product and $/$ is the fiber length.

$A=\frac{\lambda_{1}}{2 l} \cdot \sin \frac{\pi l}{\lambda_{1}} \quad L_{\varphi}=\frac{l}{2}$

Equation (4) determines the fiber density at time $t$ in any crosssection $x$ in the drawing field within the range $0<x<L-l$, and equation (5) determines the fiber density in moment $t$ between the feeding rollers. From equations (3) and (5), it follows that the density of the fiber front ends and the density of fibers in the feeding rollers grip change according to the harmonic curve and have the same wave length. The amplitude of changes in the fiber density in the cross-sections of sliver passing the feeding rollers is lower from the amplitude of changes in the density of the fiber front ends. It has been proved [1] that:

$$
A=\frac{\lambda_{1}}{2 l} \cdot \sin \frac{\pi l}{\lambda_{1}}<1
$$

The density variations in sliver cross-sections are shifted in relation to those of the fiber front ends in the phase by the angle $\varphi_{0}=\frac{2 \pi}{\lambda_{1}} \cdot \frac{l}{2}$ for $x=0$. This means that the sliver cross-section with the maximal density enters the feeding rollers grip later than the cross-section of sliver with the maximal density of the fiber front ends.

The same equation can be presented for the delivery rollers:

$$
n_{2}(t)=\frac{n_{1}}{R}\left[1+\propto \cdot \cos \left(\frac{2 \pi V_{2}}{\lambda_{2}} \cdot t\right)\right]
$$

where

$R=\frac{V_{2}}{V_{1}}$ is the average draft in the drawing zone and $\lambda_{2}=\lambda_{1} \cdot R$ is the density wave length of the fiber front ends delivered by delivery rollers.

To obtain a constant number of the fiber front ends in every cross-section of the sliver delivered, it is necessary to change draft $R(t)$ proportionally to the momentary density of the fiber front ends in the cross-section of the sliver entering the feeding rollers.

$$
n_{2}=\frac{n_{1}}{R}=\frac{n_{1}(L ; t)}{R(t)}=\text { const }
$$

The transformation of equation (8) provides the searched equation of the regulation describing a change in draft as a function of the fiber front ends density in the feeding sliver.

$$
R(t)=\frac{R}{n_{1}} \cdot n_{1}(L ; t)=\text { const } .
$$

Figure 2 presents curves $n_{1}(t), m_{1}(t), n_{2}(t)$, and $m_{2}(t)$ characterizing changes in the fiber front end density and in the fiber density before and after leaving the grip of the regulation zone rollers.

The feeding measurement device measuring the product linear density is placed in cross-section $Q-Q$. Feeding rollers are in 


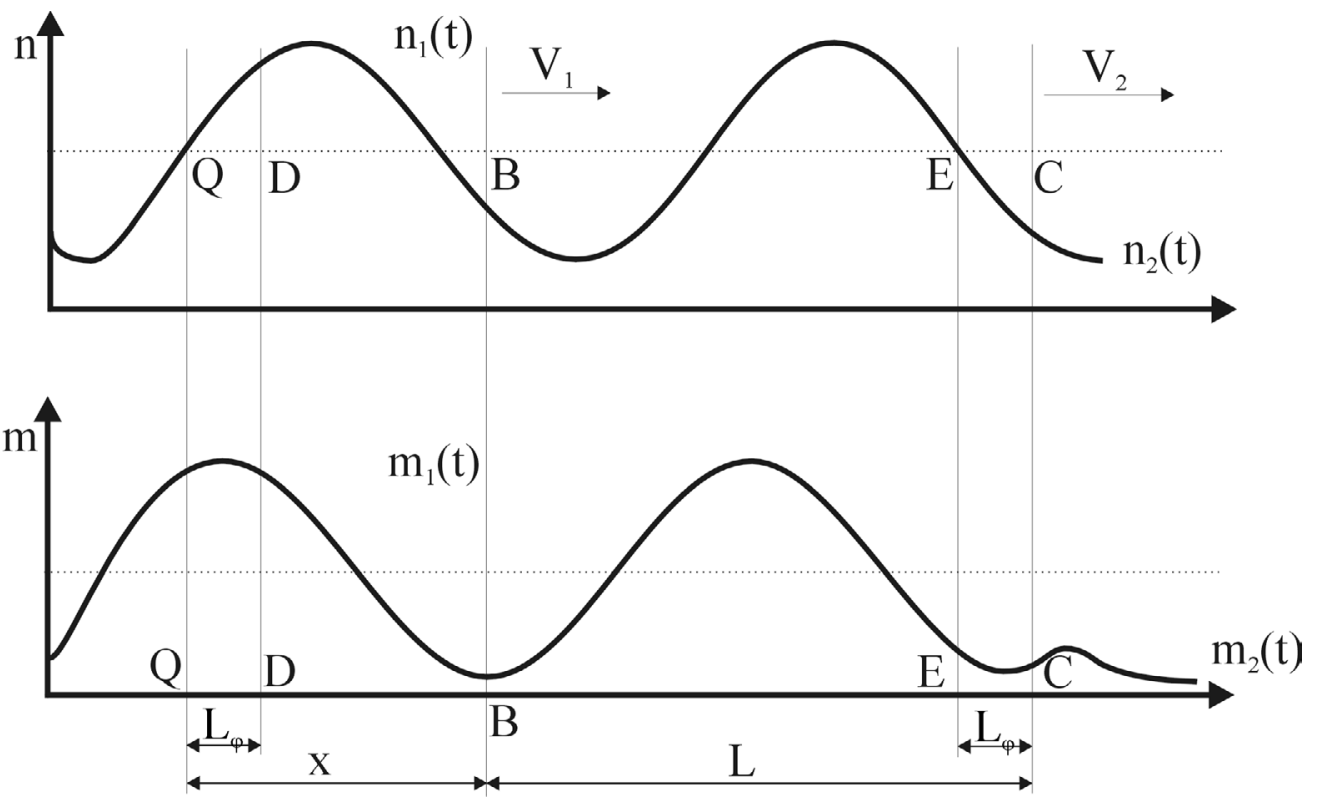

Figure 2. Curve of changes in the density of fiber ends and fiber density

cross-section $B-B$ and delivery rollers in cross-section $C-C$.

According to equations (3) and (5), in the given moment $t$ the fiber density in cross-section $Q-Q$ of the sliver present in the measuring device is equal to:

$$
m_{1}(Q ; t)=m_{1}\left[1+\alpha \cdot A \cos \left(\frac{2 \pi V_{1}}{\lambda_{1}} t\right)\right]
$$

and the density of the fiber front ends in cross-section $D-D$ shifted by $L_{\varphi}=\frac{l}{2}$ :

$$
n_{1}(D ; t)=n_{1}\left[1+\alpha \cdot A \cos \left(\frac{2 \pi V_{1}}{\lambda_{1}} t\right)\right]
$$

The fiber front ends move with a constant velocity $V_{1}$ from $D-D$ cross-section to $C-C$ cross-section and cover this route within the following time:

$$
t_{z}=\frac{L+x-\frac{l}{2}}{V_{1}}
$$

The density of the fiber front ends in cross-section $C-C$ in the given moment $t$ will be equal to the number in $D-D$ crosssection in moment $t-t_{z}$ :

$$
n_{1}(C ; t)=n_{1}\left[D ;\left(t-t_{z}\right)\right]
$$

Taking into consideration equations (5) and (13) we will get:

$$
R(t)=R\left[1-\frac{1}{A}+\frac{m_{1}\left[Q ;\left(t-t_{z}\right)\right]}{m_{1} \cdot A}\right]
$$

The equation presented is the general equation of regulation.

The mostly used form is the equation obtained with the assumption that $A \approx 1$. This assumption is correct for $\lambda_{1} \geq 6 /$. The regulation equation will then assume the following form:

$$
R(t)=\frac{R}{m_{1}} \cdot m_{1}\left[Q ;\left(t-t_{z}\right)\right]
$$

From this equation, it follows that the draft in the given moment $t$ is equal to a product of fiber density in cross-section $Q-Q$ in moment $t-t_{z}$ with a constant value of $R / m_{1}$. The fiber density or the value being its function (linear density, thickness) determined in moment $t$ in cross-section $Q-Q$ should be changed by a change in the draft or a change in velocity $V_{2}$ in cross-section $C-C$ after time $t_{z}$ that is a delay time. From equation (13), it follows that the draft should be changed when the sliver element with a specified fiber density in the measurement device is in cross-section $E-E$ in the draw field in distance $L_{\varphi}$ from delivery rollers.

The relationships presented above are binding only in the case of a product consisting of fibers with the same length. If the feeding sliver consists of fibers with various lengths, the regulation equations and the equation determining the delay times will have a bit different form.

In the case of the normal distribution of fiber length described by the equation:

$$
f(t)=\frac{1}{\sigma \sqrt{2 \pi} \cdot e}-\frac{(l-\bar{l})^{2}}{2 \sigma^{2}}
$$

the fiber density in any moment $t$ in the feeding roller grip is equal to:

$$
m_{1}(B ; t)=m_{1}\left[1+\alpha \cdot B \cos \left(\frac{2 \pi V_{1}}{\lambda_{1}} t-\varphi_{0}\right)\right]
$$

where $\bar{l}$ is the average fiber length; $\sigma$ is the standard deviation of fiber length; $m_{1}=n_{1} l$ is the average number of fibers in the feeding cross-section; $B=f\left(\lambda_{1}, l, \bar{l}, \sigma\right)$; and $\varphi_{0}=f\left(\lambda_{1}, \bar{l}, \sigma\right)$.

It has been proved that if $\frac{\lambda_{1}}{l} \geq 6$, then $B=1$, and if $\frac{\lambda_{1}}{l}<6$, then $B<1$.

The values of angle $\varphi 0$ are as follows:

$$
\varphi_{0}=\frac{2 \pi}{\lambda_{1}} \cdot \frac{l}{2}\left[1+\left(\frac{\sigma}{\bar{l}}\right)^{2}\right] \text { if } \lambda_{1} \geq 5 \text { l }
$$




$$
\varphi_{0}<\frac{2 \pi}{\lambda_{1}} \cdot \frac{l}{2}\left[1+\left(\frac{\sigma}{\bar{l}}\right)^{2}\right] \quad \text { if } \lambda_{1}<5 l
$$

Cross-section $E-E$, in the case of various fiber lengths, is a bit further from cross-section $C-C$ in the sliver compared with the previous case.

Equations (10) and (17) have the same forms, in that case the regulation equation is also correct for the product with various fiber lengths.

To control the sliver thickness, not only the measurement of linear density is needed but also the knowledge of the fiber end distribution that determines its structure. As shown by analyses $[1,4,5]$, such an information is possible to get but to a dissatisfactory extent. The function of the distribution of the fiber positions is determined on the basis of the sliver thickness function. However, it is possible exclusively for the points that are in the same distance or greater than the double fiber length. The unevenness regulation of the sliver linear density (thickness) on lengths smaller than the double fiber length is impossible to perform only on the basis of information about the sliver thickness distribution.

A quick and correct solution to the problems of automatically controlling the processes of drawing with equalizing the thickness of slivers is closely connected with the formation of mathematical models of drawing and a model of sliver depicted with fibers with different lengths.

Assuming that fibers are straightened, parallelly arranged to the product axis, and identical with respect to all their properties apart from length, one can use a model of sliver structure as a stream of fibers with identical length, whose ends are arranged on straight lines sloping to the product axis (in the case of an even product) or on curves (in the case of non-uniform product). For such a model, the density of the fiber front ends is proportional to the tangent of inclination angle of straight lines or curves. Using such a model, one can come across mathematical difficulties in the description of complex curves characterizing the fiber front ends and non-stationary drawing process, i.e. drawing a non-uniform product with a variable draft.

At present in theoretical consideration of the drawing process, one can use another model of sliver making that can be defined as "a model of continuous stream of staple fibers". For the mathematical description of this model, the following two parameters are used: the density of the fiber front ends and differential dependence of the distribution of fibers with respect to their length, $f_{n}(l, x)$, as a function of the coordinate of the sliver diameter $x$. The use of this sliver model allows one to solve many problems of drawing theory, e.g. for conditions of a nonstationary drawing, i.e. conditions that are considerably closer to the real conditions of the draw mechanism operation.

In theoretical assessment of the drawing process, one can also use the third model of sliver making with a random location of fibers. The sliver can be characterized with the use of various parameters that change on its length, e.g. the mass of sliver unit length, number of fibers, length of fibers in any sliver cross-section, degree of fiber straightening and orientation, filling the sliver cross-section with fibers, and so on. Many of these parameters are mutually connected with specified dependences. The knowledge of mutual relations between the parameters characterizing the sliver structure facilitates the formation of a mathematical model of the drawing process.

If we assume that fibers in the sliver are straightened and parallelly arranged in relation to the sliver axis, the linear density of any fiber depends only on its length while the number of fibers in the sliver cross-section is sufficiently high (the last condition allows one to use continuous quantities), then the following relations can be derived:

- for the number of fibers in the sliver cross-section:

$$
m_{t}(x)=\int_{0}^{\infty} \int_{x-l}^{x} n_{n}(x) \cdot f_{n}(l, x) d x d l
$$

- for the unit mass of sliver length:

$$
g(x)=\int_{0}^{\infty} \varsigma(l) \cdot \int_{x-l}^{x} n_{n}(x) \cdot f_{n}(l, x) d x d l
$$

where $n_{n}(x)$ is the density of the fiber front ends, i.e. the number of front ends located on the sliver section from $x$ to $x+d x ; f_{n}(l, x)$ is the differential dependence of fiber distribution with respect to their length, whose ends are located on the sliver section from $x$ to $x+d x$; and $\zeta(\mathbf{l})$ is the mass of the fiber unit length.

If every fiber cuts perpendicular to the product axis with any cross-section not more than once, then the number of fibers in the sliver cross-section is determined by the following formula:

$$
m_{t}(x)=\int_{0}^{x} n_{n}(x) d x-\int_{0}^{x} n_{\zeta}(x) d x
$$

The mutual relation between the density of the fiber front and back ends in the sliver is determined by the formula:

$$
n_{\zeta}(x)=\int_{0}^{\infty} n_{n}(x-l) \cdot f_{n}(l, x-l) d l
$$

The differential dependence of the fiber distribution with respect to the length of fibers, whose ends are located on the sliver section from $x$ to $x+d x$, is determined by the dependence:

$$
f_{\zeta}(l, x)=\frac{n_{n}(x-l)}{n_{\zeta}(x)} \cdot f_{n}(l, x-l)
$$

The differential dependence of the distribution of fibers with respect to their length that cut the cross-section of sliver with coordinate $x$ is determined by the formula:

$$
f(l, x)=\frac{1}{m_{t}(x)} \cdot \int_{x-l}^{x} n_{n}(x) \cdot f_{n}(l, x) d x
$$

So, as every fiber has its specified length and therefore it cuts several cross-sections of the sliver, the number of fibers in cross-linking $m_{t}(x)$ of sliver cannot change sharply as the density of the fiber front ends $n_{n}(x)$.

The analysis of dependence (20) shows that with $f_{n}(l, x) \neq$ const. and $n_{n}(x)=n_{n}=$ const., the sliver is uneven with respect 
to thickness, i.e. $m_{t}(x) \neq$ const. It can be shown that with $f_{n}(l, x)=$ const. and $m_{t}(x) \neq$ const., the differential dependence of fiber distribution changes $f_{\varsigma}(l, x) \neq$ const. In accordance with this parameter, the characteristic sliver structure according to the second model after the drawing process (also with a complete fiber straightening) depends on the end with which the sliver is led into the drawing mechanism.

For the third sliver model - the real sliver - all the parameters are variables and can be described with random functions. Therefore, the sliver parameters after drawing do not depend on the direction of feeding the drawing mechanism with the sliver. The analysis of the sliver model is simplified if all the dependences derived $(20 \div 25)$ are generally considered, i.e. using Laplace's methods of transformation. The transformation of the fiber front end density function into the function of fiber number in the sliver cross-section or the conveying function "number of fibers - density of front ends" has the following form:

$$
W m_{t} n_{n}(p)=\frac{M_{t}(p)}{N_{n}(p)}=\frac{1}{p} \int_{0}^{\infty} f_{n}(l) \cdot\left(1-e^{-p l}\right) d l
$$

where $M_{t}(p)$ and $N_{n}(p)$ are Laplace's transformation of functions $m_{\mathrm{t}}(x)$ and $n_{\mathrm{n}}(x)$, respectively.

The transformation of the fiber front end density into the sliver length unit mass function or the conveying function "mass of the sliver length unit - density of front ends" has the following form:

$$
W g n_{n}(p)=\frac{G(p)}{N_{n}(p)}=\frac{1}{p} \int_{0}^{\infty} f_{n}(l) \cdot \varsigma(l) \cdot\left(1-e^{-p l}\right) d l
$$

where $G(p)$ is the Laplace's transformation of function $g(x)$.

The use of the research method of the drawing process as a spatial-time scheme and the second structural model of sliver under diminishing its thickness considerably extend the opportunities of a profounder analysis of the process.

\section{Automatic draft regulation - analysis of the model in modified regulation systems}

The analyses of short-term regulation systems carried out for many years [7-10] have shown that the sliver linear density levelness equalized out by the short-term autoleveller is a function of time. In slivers, retarding processes occur, whose duration decreases with increasing average fiber length and increasing nominal draft in the regulator. The results of the tests performed have confirmed the necessity of constructing a short-term autoleveller whose algorithm of operation will take into account the retardation phenomena of slivers.

Equation (15) describes the operation of a typical system of short-term regulation. In a mechanical measuring device of the draft regulator, the cross-section of fiber stream is a rectangle with a constant base and variable height dependent on the thickness of the passing sliver. Proportional relationship between these quantities leads equation (15) to the following form:

$$
R(t)=\frac{R}{g_{1}} \cdot g_{1}\left(Q ; t-t_{z}\right)
$$

where $g_{1}\left(Q ; t-t_{z}\right)$ is the momentary fiber stream thickness in the measuring cross-section $Q$.

There is known retardation equation $r$ of sliver after the shortterm regulation [8]:

$$
r=a \cdot(R-k)^{b} \cdot e^{c \cdot(R-k)}
$$

where $a, b$, and $c$ are shape coefficients depicting changes in the retardation curve and $k$ is the limiting draft with no retardation.

Changes in the draft in regulation zone force changes in retardation $r(t)$ of particular regulated sliver sections, i.e.:

$$
r(t)=a \cdot[R(t)-k]^{b} \cdot e^{c \cdot[R(t)-k]}
$$

The regulated sliver retardation depends on the momentary draft in regulator that occurred when the given section was in the regulation zone. This concerns the sliver sections that were thinned with a draft greater than $k$ (limiting draft with no retardation). From equation (29) it follows that the sliver sections drawn with draft $R_{r \max }=-b / c$ undergo the highest retardation.

As the retardation phenomenon is adverse for the distribution of sliver linear density equalized by the short-term autoleveller, while it is an unavoidable element of the equalizing the stresses in fibers, this phenomenon should be taken into account in the algorithm of regulator operation. The sliver should be readjusted in such a way that through the use of appropriately higher momentary drafts it could be possible to account the retardation phenomenon that occurs later.

The new algorithm of regulator operation should be expressed by the equation:

$$
R^{\prime}(t)=R(t)+R(t) \cdot r(t)=R(t) \cdot[1+r(t)]
$$

After taking into account equation (30), the new algorithm of regulator operation has the form:

$$
R^{\prime}(t)=R(t) \cdot\left\{1+a \cdot[R(t)-k]^{b} \cdot e^{c \cdot[R(t)-k]}\right\}
$$

The only variable in equation (32) is $R(t)$, i.e. according to equation (28) the value of $g_{1}(Q ; t-t z)$, and the determination of the remaining parameters should proceed in the following order:

- for the given linear density of feeding sliver, define nominal draft $R$ in the regulator (or indirectly the average linear density of delivered sliver), then calculate the value of $g_{1}$, i.e. the average thickness of feeding stream;

- $\quad$ based on the type of processed fibers, establish draft $R_{r \max }$, with which retardation reaches the maximal value, and the 
value of parameter $k$, i.e. the limiting draft with which the sliver retardation does not occur; and

- for the given nominal draft in regulator and type of processed fibers, establish the values of coefficients $a, b$, and $c$ of the equation, taking into account relation $R_{r \max }=-b / c$.

The proportional dependence of regulating draft on the voltage signal initiating the motor operation of the draw roller drive enables the presentation of equation (31) in the following practical form:

$$
U^{\prime}(t)=U(t) \cdot[1+r(t)]=U(t) \cdot\left\{1+a[U(t)-k]^{b} \cdot e^{c \cdot[U(t)-k]}\right\}
$$

The change in the algorithm of draft regulator operation should take into account the controlling voltage signal with momentary value $U^{\prime}(t)$ that changes standard signal $U(t)$ according to equation (33). The realization of the new algorithm must be carried out digitally on the basis of the controlling signal correction system, which forces the variability of many process parameters. The regulation performed in this way will take into account the sliver retardation phenomena and make it possible to increase the evenness of slivers feeding successive machines in the technological process. As a result of this, the evenness of yarn will be increased contributing to an improvement in the quality of flat textile fabrics.

The validation of the presented model of the fiber movement in the drawing mechanism field, aiming at establishing a regulation equation with a modified operation algorithm of the short-term draft regulator of slivers made of staple fibers, is presented in the author's publications [7-10], cited in the literature review.

\section{References}

[1] Czekalski J. (2014). Modelowanie procesu rozciągania strumienia włókien w aparatach rozciągowych przędzarek. Zeszyty Naukowe PŁ, nr 1185.

[2] Rutkowski J. (2011). Tenacity of cotton yarns joined during the rewinding process. Fibres \& Textiles, 19(1).

[3] Taher Halimi, M., Ben Hassen, M., Azzouz, B., Sakli, F. (2007). Effect of cotton waste and spinning parameters on rotor yarn quality. Journal of the Textile Institute, No. 5.

[4] Min, W. (2005). Function and characteristics of autoleveller draw frame. Fibres \& Textiles in Eastern Europe, No. 5.

[5] Baumann, M., Heitmann, U., Planck, H. (2008). Reduzierung der Faserbeanspruchung und Kardierkräfte beim Hochleistungskardieren. Melliand Textilberichte, No. 1-2.

[6] Sevostianov, A. G. (1991). Vyravnivanie tekstilnych materiałov. Izviestia Vysszych Učebnych Zaviedienij. Technologia Tekstilnoj Promyszliennosti nr 6.

[7] Idzik M. (2006). Analysis of work of a card autoleveller with a new algorithm of operation. Fibres \& Textiles, 14(2).

[8] Idzik, M. (2000). Optimization of work parameters of a short-term autoleveller. Fibres \& Textiles in Eastern Europe, 8(4).

[9] Idzik, M. (2006). Assumptions for constructing an autoleveller with a new algorithm of operation. Fibres \& Textiles in Eastern Europe, 14(1).

[10] Idzik, M. (2003). Effect of operating a control system on linear density distribution of a fibre stream. Fibres \& Textiles in Eastern Europe, 11(1). 